\title{
The treatment of canine mast cell tumours with electrochemotherapy with or without surgical excision
}

\author{
R. Lowe1, A. Gavazza2, J. A. Impellizeri3, D. M. Soden4 and G. Lubas2 \\ 1 Ashleigh Veterinary Clinic Limited, Knaresborough, UK \\ 2Department of Veterinary Sciences, University of Pisa, Pisa, Italy \\ 3 Veterinary Oncology Services, New York, NY, USA \\ ${ }_{4}$ Cork Cancer Research Centre, University College Cork, Cork, Ireland
}

\section{DOI: $10.1111 / \mathrm{vco} .12217$}

\begin{abstract}
To describe the results of electrochemotherapy (ECT) in dogs with mast cell tumours (MCTs) either as first line therapy or as an adjuvant to surgery. The treatment combines administration of lowdose chemotherapeutic drugs with the application of microsecond electric pulses, which cause the temporary permeabilization and increased porosity of the tumour cell membranes. The design of this study is a retrospective case series. A total of 51 dogs with MCTs were included and classified according to ECT procedure into 4 groups (ECT only, 15 cases, intra-surgery ECT, 11, ECT Adjuvant to surgery, 14 , Surgery followed by ECT, 11). The four groups (staged with location, size and grade) were evaluated to assess complete or partial remission, disease free interval, overall survival time and local toxicity. In this case series, Boxers, mixed breed and Labrador Retrievers, male dogs, between 4 and 9 years old were more represented. MCTs were predominantly grade 2 (Patnaik) and T stage $0-1,1-1$ (World Health Organization). Treated lesions were most commonly identified on the hindlimb and head where curative surgery would involve cosmetic or functional compromise. The intra-surgery group of dogs showed the best disease free interval with Kaplan-Meyer analysis. Local toxicity induced by ECT ranged mostly from 1 to 4 in a 5-point arbitrary scale with 0 - no toxicity to 5 - highest toxicity. Inthis study, ECT canbe appliedsuccessfully as anexclusive therapy insmaller MCTs as an alternative to surgery. ECT can be combined with surgery either intra-operatively or post operatively for larger lesions without significant toxicity. Keywords

comparative oncology,

electrochemotherapy, large

animal, mast cell tumours, oncology
\end{abstract}

Introduction

Electrochemotherapy (ECT) is clinically established in Europe for cutaneous local cancer

treatment.1,2 It combines the intralesional and/or systemic administration of speci!c chemotherapeutic drugs (bleomycin or cisplatin) with permeabilization of the cell membrane by the application of electric pulses. "e electropermeabilization or electroporation of the cells in the tumours induces an electrically mediated reorganization of the plasma membrane allowing the chemotherapeutic to be absorbed via passive di\#usion. ECT can be used to treat cutaneous and subcutaneous tumours (e.g., sarcomas, carcinomas, 
melanoma and mastocytoma) regardless of histological type.3-6

A recent clinical review of ECT-treated human tumours (total of 1894 cases from 44 studies), con!rmed that ECT had signi!cantly higher e\#ectiveness than chemotherapy with bleomycin or cisplatin alone."is was signi!cantly higher for intratumoural than for intravenous administration of bleomycin. ECT was also more e\#ective in human sarcomas than in melanoma or carcinomas. 7

MCTs are among themost commonly diagnosed dermatological neoplasms in the dog, accounting for $7-21 \%$ of all skin tumours and for $11-27 \%$ of all cutaneous malignancies. 8 Local control of these tumours can be challenging and recurrence rates range from 22 to $54 \%$ a\$er surgical excision. 8 Adjuvant treatments commonly include chemotherapy (prednisone, vincristine, vinblastine, lomustine or combinations), radiation therapy and receptor

tyrosine kinase inhibitors such as masitinib or toceranib.8-10

In most studies implementing ECT, bleomycin and cisplatin were the preferred chemotherapies used in small animals with bleomycin injected IV or intratumourally followed by application of electric pulses. 2 Cisplatin injected intratumourally was tested in several clinical trials by Tozon et al.11 Both drugs can be used in ECT but bleomycin is easier and safer as IV administration, which may be more practical in di\#use or larger tumours. In one study of 28 dogswith incompletely excised MCTs treated with ECT utilizing bleomycin, a high response rate with local tumour control, was observed (about 85\%).12 In a second study from almost the same group of authors, included 37 dogs with incompletely excised MCTs treated with 
ECT utilizing cisplatin. A good local response rate was observed (about 62\%).13 In both studies several advantages were clear including low cost, limited toxicity and ease of administration. "e major limitation was the lacking of a control group and the treatment of grade $2 \mathrm{MCT}$ according to Patniak, which are considered to be highly curable with appropriate surgical excision only.13

"e clinical side e\#ects of ECT treatment in MCT are minimal. During the application of electrical pulses, muscle contractions were observed only during electric pulse delivery. Locally, resolution of the tumour is noticed during the !rst week, with the development of a super!cial scab in the following 2-4 weeks and !nally detachment of this layer in the next 5-8 weeks. One possible side e\#ect reported when treating MCTs with ECT could be degranulation of tumour cell: using cisplatin one study did not report any local or systemic side e\#ects of this type,13 another reported this side e\#ect in $32 \%$ of cases treated.14 When using bleomycin, a study reported a $7 \%$ side e\#ect. 12 In our retrospective multi-institutional study during a period ranging from 2004-2014, the combination of ECT with bleomycin in dogs a\#ected byMCT, either as !rst line treatment or as adjuvant to surgery or post-surgery relapse, is described. "e advantages and the side e\#tects, along with the follow-up of the patient are reported.

\section{Materials and methods}

A total of 51 dogs with MCT diagnosed either with cytology, histology or both techniques were included. Most of patients were purebred breeds (only 8/51 mixed), both sexes including neutered were represented with a slight prevalence of males (24) in comparison with females (17) and the age 
range was 2 to 15 years. Details of their signalment (breed, age and sex) are reported in Table 1.

Patients were staged depending on the initial size of the tumour, the anatomic location, !rst or second opinion exam and owner's consent to the procedure. All patients underwent at least cytology of the mass, cytology of the regional lymph node's

draining from the area of the primary MCT (if palpable) and a minimum laboratory database (complete blood count, serum or plasma biochemical pro!le including total protein, albumin, urea, creatinine, total bilirubin, alanine transaminase, aspartate transaminase, alkaline phosphatase, gamma-glutamyl transferase, glucose, cholesterol, calcium, phosphorus, prothrombin time, partial thromboplastin time). Diagnostic imaging (3-view thoracic radiographs and abdominal ultrasound) was performed if necessary to stage patients with the largest MCTs (generally with tumour size over $3 \mathrm{~cm}$ ) or with concurrent abnormalities fromother organ impairment established on the minimal laboratory data previously described.

Surgery and/or radiotherapy were discussed as treatment options with the owners. If rejected, ECT was discussed and the informed consent was obtained in all cases. In the case when incomplete surgery was already done, the addition of ECT was o\#ered as an adjunct treatment for local control.

"e main criterion of patient inclusion was that the surgery was not the best procedure because it would be inadequate or the e\#ects of surgery would have been unacceptable for function and

Table 1. Signalment summaries for breed, age and gender of 51 dogs included in the case-load

\section{Breed \# Age (yy) \# Sex \#}

Boxer $1122 \mathrm{~m} 24$ 
Xbreed 845 mn 10

Labrador Retriever 756 fn 9

SBT $568 \mathrm{f} 8$

Golden Retriever 477

Bernese MD 285

JRT 297

Springer Spaniel 2102

Beagle 1114

Border Collie 1123

Boston Terrier 1131

Cocker Spaniel 1151

Dobermann 1 Median age 7.0

Pug 1

Weimeraner 1

WHWT 1

Yorkshire terrier 1

Xbreed, mixed bred; Bernese MD, Bernese Mountain dog; JRT, Jack

Russell Terrier; SBT, Stafforshire Bull Terrier; WHWT, West

HighlandWhite Terrier; \#, number of dogs; $m$, male; $m n$, male neutered;

$\mathrm{fn}$, female neutered; $f$, female; yy, years.

Statistics between the four treatment groups for differences by breed

using the Fisher's exact test was not significant $(P>0.05)$. In

addition, the same statistic in evaluation for group differences in age and

gender using the Chi square analysis was not significant

$(P>0.05)$.

Table 2. Dogs with MCT included in the case list sorted by district, type of

ECT treatment, site and size of MCT

District a ECT alone (15) ECT intra-op (11) ECT post-op (14) ECT recur (11) Head (15) Nose (4), face (3), ear

$$
\text { (1), lip (1) }
$$

Ear (1) Nose (1), face (1),

conjunctiva (1)

Nose (1), lip (1)

Body-front (4) NA NA Thorax lateral $(1 \times 3)$

and ventral (1)

Neck (1), thorax $(1 \times 2)$ 
Body-rear (7) Tail $(1 \times 2)$ Prepuce (1) Ischial (1), anus $(1 \times 5)$ Ischial (2), anus (1)

Forelimb (6) Antebrachium (1),
elbow (1), carpus

$(1 \times 2)$

Elbow $(1 \times 2)$ Carpus (2) NA

Hindlimb (19) Tarsus (1), paw (1) Tarsus (3), hock (2),

stifle (2), thigh (1)

Popliteal (1), hock

$(2 \times 2)$, paw (2)

Thigh $(1+1 \times 2)$, hock

(1), paw (1)

Sizeb

Mean 0.741 .236 .264 .01

Median 1.002 .802 .254 .10

Range 0.3-3.0 0.7-6.0 0.5-32.0 0.6-12.0

For each site in brackets the number of cases is reported; NA, not applicable; $(1 \times 2)$, in this site two tumour mass; $(1 \times 3)$, in this site three tumour mass; $(1 \times 5)$, in this site five tumour mass.

aStatistics between the four treatment groups for district comparison using Chi square was not significant $P>0.05$.

bSize tumour comparison by mean of Kruskal-Wallis $P=0.003$ was

significant and the Dunn'sMultiple comparison test supported the significant level between the ECT alone group versus the ECT recur group.

appearance. ECT treatment was divided into four di\#terent categories (see Table 2).

(1) ECT sole (ECT alone) therapy (15 cases) performed

in smaller MCTs (mean and median size: $1.39 \mathrm{~cm} ; 1.00 \mathrm{~cm}$ ) - these MCTs were clearly visible macroscopically and could be resected by conventional surgery but the owners elected to pursue ECT.

(2) ECT intra-operative (ECT intra-op) (11 cases), performed in larger MCTs (mean and median size: $2.71 \mathrm{~cm} ; 2.80 \mathrm{~cm}$ ) in comparison 
with the previous group, during the surgical cyto-reduction of the MCT; the choice to treat these dogs with a combination of surgery and ECT was based on the fact that ECT only could not cover all the tumour and the complete control could be obtained

by surgery and ECT; indeed the majority of the tumour was excised, with no attempt on obtaining complete margins (as standardly accepted of 2-3 cm), and ECT was applied to lateral and deep margins before wound closure; in some cases macroscopic tumour was still present and histologically the margins were showing in! Itration of mastocytes; this method was used where radical excision would have resulted in functional or cosmetic compromise due to anatomic limitation.

(3) ECT adjuvant to surgery (ECT post-op) (14 cases), where the surgical cyto-reduction of the MCT was initially performed attempting wide margins but where histological examination showed inadequate margins ECT was applied a\$ser a period of 2-4weeks (range 13-29 days)."e reason for the delay

was to allow ECT treatment as early as possible a\$ser adequate wound strength had developed a\$ser surgery (at surgeon discretion).

"e ECT adjuvant was carried out on post-surgical scar-lines and their margins of about 1-1.5 cm (maximum length of the scar $32 \mathrm{~cm}$; mean and median size: $6.26 \mathrm{~cm}$;

$$
2.25 \mathrm{~cm} \text { ). }
$$

(4) ECT performed a\$er surgery at the recurrence of MCT, which was macroscopically visible in the same site of initial surgery 
(ECT recur) (11 cases); ECT was carried out in these patients a\$ser a greater period than 1 month from prior surgical treatment.

"e mean long-axis dimension was $4.72 \mathrm{~cm}$ (mean and median size: $4.73 \mathrm{~cm} ; 4.10 \mathrm{~cm}$ ). "e several sites and the size of MCT (presented as mean, median and range for each group) are reported in Table 2 . In nine patients there were multiple mast cell tumours at the presentation.

MCTs were graded by di\#erent pathologists using the Patnaik histology grading scheme.15 Also

the World Health Organization (WHO) clinical staging16,17 (Tables 3 and 4) was used retrospectively.

Grade 2 tumours were also graded by the mitotic index (MI) (number of mitotic !gures per 10 high power !elds) and all cases in this study had $\mathrm{MI}<5.18$

Table 3. Staging of MCT according to Patniak score of dogs included in the case-load

\section{Patniak \\ ECT alone \\ ECT intra-op \\ ECT post-op \\ ECT recur Total}

Grade 141005

Grade 2111014944

Grade 300022

Total 1511141151

Statistics for differences between the four treatment groups for differences in stagingMCT according to Patniak score using Chi square was significant ( $P=0.022)$.

Table 4. Staging of MCT according toWHO clinical score 
of dogs included in the case-load

T-stageWHO

ECT

alone

ECT

intra-op

ECT

post-op

ECT

recur Total

0-1 1014722

I-1 1480022

II-1 010001

II-2 000022

III-1 020013

III-2 000011

Total 1511141151

Statistics for differences between the four treatment groups for differences in staging MCT according to WHO clinical score using Chi square was highly significant (P!0.0001).

"e electroporation was performed using three di\#erent generators whose electrical output was identical as con!rmed by oscilloscope analysis [Cytopulse PA4000 (Cyto Pulse Sciences, Glen Burnie, MD, USA) for 13 cases; Cytopulse Oncovet (Cyto Pulse Sciences) for 35 cases; and Cliniporator (IGEA S.p.a., Modena, Italy) for 3 cases.].

Needle electrodes [Gehl Needle electrodes, (Cyto Pulse Sciences)] of 1 or $2.5 \mathrm{~cm}$ length were used for delivery of the electrical pulses. "e pulse pattern employed was 8 monophasic square pulses of $0.1 \mathrm{~ms}$ each at a frequency of $1 \mathrm{~Hz}$ with the !rst generator [Cytopulse PA4000 (Cyto Pulse Sciences) for 13 cases]. "e frequency used with the second generator [Cytopulse Oncovet (Cyto Pulse Sciences) for 35 cases] was $5 \mathrm{kHz}$ in 23 cases 
and $1 \mathrm{~Hz}$ in 11 , while all three patients with the third generator [Cliniporator (IGEA S.p.a.) for 3 cases.] were treated at a frequency of $5 \mathrm{kHz}$. Overall, the $1 \mathrm{~Hz}$ frequency was used in 25 patients, the remaining 26 receiving $5 \mathrm{kHz}$. "e needle electrode [Gehl Needle electrodes, (Cyto Pulse Sciences)] pattern consists of two parallel $1-1.5 \mathrm{~cm}$ rows of 6 needles, the rows being $6 \mathrm{~mm}$ apart. "e pulse amplitude was 600-720 volts (1000-1200 volts $/ \mathrm{cm}) .19,20$ In the case of the Italian equipment [Cliniporator (IGEA S.p.a.) for 3 cases], di\#erent electrodes shapes (hexagonal or linear, with each 8 needles) and $20 \mathrm{~mm}$ length needles were used. "e voltage setting for the Cliniporator was pre-set by the manufacturer at 1000 volts/cm with current varying between applications depending on tissue conductivity (safety limit of 20A).

For all ECT procedures, Bleomycin was administered intravenously at a dose of 15000 (! \$een thousand)

IU per square metre of body surface area. 5 ASer a delay of $8 \mathrm{~min}$ to allow the drug to enter the interstitial spaces and equilibrate, electroporation was applied to the site via standard operating procedure. 21

Treatment areas were prepared as for surgery. Skin was clipped and aseptically prepared with povodine-iodine wash (10\% iodine). In dogs receiving ECT alone, the tumour, plus a $1-2 \mathrm{~cm}$ margin in all planes into grossly normal tissue was accessed by penetration of the electrode needles. "e treatment was started in the margins and progressed concentrically into the tumour mass, thus avoiding transference of tumour cells from the centre to the margins. Similarly, when ECT was used intra-operatively, a $1-2 \mathrm{~cm}$ margin in all directions was treated. In dogs treated with ECT adjuvant to 
surgery, where there was no macroscopic disease, the treatment volume extended at least $1.5 \mathrm{~cm}$ from the healed excision site in all planes except in cutaneous MCTs where $0.5 \mathrm{~cm}$ depth was used.

"e treatment was completed within 20min, which is the window of time used to optimize tissue drug concentration. Some larger tumours required more time (i.e., 35 min for a case of ECT adjuvant to surgery along a scar-line of about $32 \mathrm{cmusing} 1 \mathrm{~Hz}$ ). All dogs were treated under general anaesthesia (premedication with Medetomidine/Butorphanol, induction with Alfaxalone, maintenance with iso\%urane/O2/N2O, and reversal of Medetomidine with Atipamezole). In addition, all patients received perioperative and postoperative analgesia either NSAID, opioid or both (Butorphanol at $0.1 \mathrm{mg} / \mathrm{kg}$ SC BID and/or Meloxicam $0.2 \mathrm{mg} / \mathrm{kg}$ daily per os in the !rst 5-7 days post-treatment). Antibiotic coverage was given to all patients during the period of tissue necrosis: either Amoxycillin/Clavulanate, $10 \mathrm{mg} / 2.5 \mathrm{mg}$ per kg BID per os or Clindamycin $15-37 \mathrm{mg} / \mathrm{kg}$ daily per os.

Patients were assessed at varying intervals every 2-5 days in the !rst 2weeks a\$ er treatment either by the authors ( $R L$ or $G L$ ) or by the referring veterinary surgeon and approximately every 2 weeks ( $R L$ or $\mathrm{GL}$ ) until healing was complete.

"erea\$er, progress was monitored at intervals until at least 2 years either by examination of the patient or by telephone contact with the owner. "e progression of changes in the treated tissue was recorded by digital camera images. A grading score (5-point scale) for tissue necrosis was established by authors as follow using the photographic records of patients: $0=$ none, $1=$ slight swelling, $2=$ swelling/necrosis $<1 \mathrm{~cm}, 3=$ severe swelling, 
4=deep necrosis and 5=severe swelling and tissue loss.

No clinico-pathological monitoring was considered necessary. Myelosuppression has not been reported a\$ser Bleomycin therapy in human or dog, only drug-induced lung injury has been reported a\$er prolonged use in humans.22,23 None of the dogs treated had any clinical signs of respiratory

pathology.

"e responses were evaluated as complete remission

(CR) or partial response (PR) (considered as at least $50 \%$ reduction in tumour size) obtained in the cases enrolled in groups ECT alone and ECT recur where MCT was clearly visible macroscopically.

In the other two groups, ECT intra-op and ECT post-op where the ECT was used to enhance the antineoplastic action against MCT that could bemicroscopically present. In these two groups, the same responses as above were used ( $C R$ as no reappearance of the MCT, PR as reappearance of the tumour with a minor size in comparison to the !rst occurrence).

"e disease-free interval (DFI) was calculated from the date of treatment with ECT to the !rst recurrence or death of the animal unrelated to the MCT disease; or alternativelywhenwe censored the length of DFI at the date we arranged this paper (in the beginning of 2014). "e overall survival time (OST) was calculated from the date of treatment with ECT to the date when the patient died or when we arranged this paper (in the beginning of 2014).

"e results were subclassi!ed by ECT modalities into four groups. Parameters analysed within each group were: (1) signalment of dogs enrolled including breed, sex, and age; (2) staging of MCT including site; (3) mean tumour size (in cm); (4) Patnaik 
and WHO classi!cation; (5) outcome of the treatment as number of dogs having CR or PR, DFI and OST; (6) evaluation of local toxicity observed; (7) relationship, if any, between CR or DFI and size of the tumour; (8) relationship, if any, between Patniak

or T-stage WHO and CR or DFI; (9) relationship, if any, between the size of the tumour and toxicity seen.

Statistics

Statistical tests were analysed using appropriate so\$ware (MedCalc® version 14.8.1.). In detail: Fisher's exact test for breed; Chi-squared test for sex, age, Patnaik and WHO classi!cation, site or region of $\mathrm{MCT}$, local toxicities grading; D'Agostino and Pearson test for normal distribution; KruskalWallis, and Dunn's multiple comparison test for tumour size; Kaplan-Meier curve and Logrank test for DFI and OST. Relationships as described above were studied with linear and non-linear !tting and Kruskal-Wallis test if necessary.

\section{Results}

Table 1 identi!es breeds, age and gender group of dogs enrolled. Among breeds Boxers,mixed-breeds and Labrador Retrievers were more represented in all groups. "e statistical di\#erence by breed between the four groups evaluated with Fisher's exact test was not signi!cant. "e age of several groups ranged from 6 to 9 years old (median 7 years). "e statistical di\#erence by age between the four groups evaluated with Chi-square analysis was not signi!cant. Males and neutered males in comparison with females and neutered females were more represented. "ere was no statistically signi!cant di\#erence (Chi-square analysis) in the distribution of gender between treatment groups. Table 2 shows the anatomic region, type of treatment 
and size (reported as mean, median and range) for each group of dogs with $\mathrm{MCT}$ treated with ECT. "e ECT alone group included most patients with tumours on the head region, while in the ECT intra-op group, the hind-limb location was more represented. "e statistical di\#erence by region between the four groups evaluated with Chi square analysis was not signi!cant. "e size of the MCT tumour between the four groups evaluated with Kruskal-Wallis analysis was statistical significant $(P=0.003)$. "e Dunn's Multiple comparison test demonstrated the statistical signi!cant level between the ECT alone group versus the ECT recur group. No relationship was demonstrated by linear or non-linear !tting between the size of the tumour and CR or DFI.

Table 3 identi!es the grading scheme according to Patnaik. Grade-2 MCTs were more represented and the statistical di\#terence by stage between the four groups evaluated with Chi Square analysis was signi!cant $(P=0.022)$. AllMCTs had a $M I$ less than 5 . No relationship was demonstrated by non-linear !tting followed by Kruskal-Wallis test between this grading scheme and CR and DFI.

Table 4 shows the staging according to theWHO clinical score of dogs enrolled and sorted as in previous tables. T-Stage $0-1$ and I-1 MCTs were more represented and the statistical di\#erence by stage between the four groups evaluated with Chi square analysis was highly signi!cant $(P<0.0001)$. No relationship was demonstrated by non-linear !tting followed by Kruskal-Wallis test between this grading scheme and CR and DFI. Among the four groups, dogs with $C R$ and $P R$ was as following: ECT alone $12 \mathrm{CR}(80 \%)$ and 3 PR (20\%) (these three dogs underwent to surgery 
procedures); ECT intra-op 10 CR (91\%) and 1 PR (9\%) (the dog underwent to surgery procedure and ECT a\$er 20 days); ECT post-op 13 (93\%) CR and 1 PR (7\%) (the dog also developed several other MCTs in di\#terent region and underwent to surgery procedure); ECT recur 7 CR (64\%) and 4 PR (36\%) (these four dogs had previously surgery and ECT was their last chance to control the recurrence of MCTs in the initial site).

Table 5 de!nes the local toxicity in the four treatment groups. "e local toxicity score ranged mostly from 1-4 with the lowest scores for most dogs belonging to the ECT recur group (as shown also by the median). "e statistical di\#erence by local toxicity between the four groups evaluated

Table 5. Local toxicity between groups

\section{Local toxicity}

ECT

alone

ECT

intra-op

ECT

post-op

ECT

recur Total

001214

1413513

2414110

3534214

424129

501001

Median 2321 -

Statistics between the four treatment groups for differences in local toxicity using Chi square was not significant $(P>0.05)$.

Figure 1. Kaplan-Meyer graph of the four group of dogs withMCT showing the DFI time (days) - the comparison of 
survival curves with the Log-rank test was $P=0.022$.

with Chi square analysis was not signi!cant. No relationship was demonstrated by non-linear !tting followed by Kruskal-Wallis test between the local toxicity and size of MCTs into the four groups. No speci!c treatment other than local care of the scar and antibiotics was necessary in all patients. No evidence of degranulation from the tumour was observed and the swelling of the treated area was not greater than other type of tumour treated with ECT by the authors (i.e. !brosarcoma or squamous cell carcinoma).

Fig. 1 shows the Kaplan-Meier graph showing the DFI time (days)."e statistical comparison of survival curves with the Log rank analysis was signi!cant $(P=0.022)$ with the ECT post-op treatment group with the best results in terms of days with complete remission. Data were censored at the beginning of 2014.

Fig. 2 is the Kaplan-Meier graph showing the OST to censoring, either by latest report or death.

Figure 2. Kaplan-Meyer graph showing the OST to censoring, either by the latest report or death."e statistical comparison of survival curves in OST with the Log-rank analysis was not signi!cant."e comparison of survival curves with the Log-rank test was $P=0.255$.

"e statistical comparison of survival curves inOST with the Log rank analysis was not signi!cant. Data were censored at the beginning of 2014

\section{Discussion}

Curative surgery for canine MCT may involve cosmetic or functional compromise or aggressive extirpation, for example, amputation. Of course, surgery remains the mainstay of treatment inMCT. In this study, we utilized the application of electrochemotherapy in di\#terent clinical scenarios of 
MCTs to improve the results against this tumour. We adopted the best treatment according to the owner consent for each case using a combination of ECT alone, ECT used simultaneously to surgery (intra-operative, ECT intra-op), ECT a\$ser a certain time post-surgery to prevent a relapse (adjuvant, ECT post-op) or !nally when surgery was already done with failure to controlMCT and then ECT was applied (ECT recur). One limitationwas the lack of a control group of dogs (i.e. treated with surgery only or treated with adjunctive chemotherapy) for each of the four types of treatment. Once owners elected $E C T$, it was unethical, as an unfunded study, to not provide the available treatment.

In general, the four groups were showing almost the same distribution in terms of breed (Boxer, mixed breed and Labrador Retrievers were more represented), gender (males were predominantly

represented in all groups) and age (median age was ranging from 6 to 9 years old). "is distribution is in agreement as reported in a textbook.16 "e two di\#erent grading used for MCT staging shows that grade-2 for Patnaik and T-stage 0-1 and I-1 for WHO were more represented in dogs included in this report.

"e regions of MCT treated were di\#terent for each group, especially for the ECT alone group (more head sites) and the ECT intra-op group (more hindlimb sites)."e site of MCTs in the four treatment

groups was di\#erent for the ECT alone group tumours were located mostly in the nose and face. In the ECT intra-op group most were located on the tarsus. In the ECT post-op group, several MCTs were located on or near the hock. In the ECT recur group,MCTs were mostly located in the ischial and thigh regions."e size of MCTs treated varied and 
were statistically signi!cant between the ECT alone group (smaller size) and the ECT recur group (larger size). "e ECT post-op group was highly heterogeneous in size as seen in the highest di\#erence between the mean and median tumour size. "ere was no relationship between $\mathrm{CR}$ and/or $\mathrm{CR}$ and/or

DFI and tumour size."e comparison between the groups was challenged as a few MCTs were multiple on the same pet ( 7 cases had 2 tumours, 1 case each had 3 or 5 tumours, respectively). "e cases with multiple MCTs did not show any di\#erence in tumour response rate and included in the analysis with Kaplan-Meier curves.

Local toxicity was minimal ranging mostly from 1 to 4 (on a scale from 0 to 5)."e ECT recur group showed the least local toxicity as shown by median. "ere was not any relationship between the size of the tumour and the toxicity seen.

"e best scores for CR were found for ECT post-op and ECT intra-op group (respectively 93 and $91 \%$ of CR), then ECT alone group ( $80 \%$ of CR) and !nally ECT recur group (64\% of CR).

"e DFI in days with complete remission identi !ed the ECT post-op group with the best score, while the di\#erent treatments were not in\%uencing the OST.

In practice, most of MCTs originating from dermal are tumours of grade 1 or 2 using Patnaik score and can be cured with surgery alone, provided the site is amenable to adequate surgical resection.8,16

In addition, the quality of the deep margin is as important as that of the lateralmargins to guarantee complete extirpation even with some controversy about the importance of securing cleanmargins.9,16 With MCTs of distal extremities, where complete excision of a lowor intermediate grade tumour 
cannot be achieved, four therapeutic options are standard: amputation, external-beam radiotherapy, a combination of the two previous procedures and !nally surgery and chemotherapy.16 ECT is a reasonable

option to be added to this list, when surgery cannot guarantee an adequate resection with wide margins. In our study, the distal extremities, where a loss or reduced functionwas possible if only surgery was applied, represented about $50 \%$ of the cases treated (25/51) and MCT that were located on the head equaled about $30 \%$ (15/51) where a cosmetic

reason was considered to not pursue surgery.

ECT in our opinion is a valuable therapeutic in the armamentarium of treatment options against canine MCT.23,24 We have shown that options exist to e\#tectively treat canine MCT with ECT depending

on their size and anatomic location as it relates to maintaining a functional patient and avoiding, in some cases extreme surgery such as amputation. While the lack of a control group is a limitation, the results support the statistical signi!cance of the use of ECT for the local control of canine MCT is several clinical settings.

Future studies could evaluate the molecular pattern of proliferationmarkers and c-kit expression as they relate toMCT behaviour when ECT is a therapeutic involved in local control; as well as the newer

2-tier system for grading. 25,26

An additional bene!t of electroporation relates to its stimulation of both an adaptive and innate immune cell in! Itration into treated tissues in the days post procedure. 27 Previous data have demonstrated a large in!ltration of dendritic antigen presenting cells into the ECT-treated tumour tissue post procedure along with a local increase in CD8+ T cells, 27 which is a positive prognostic indicator 
for long-term survival according to the most recent clinical immunological data.28,29

Used in combination with immunomodulatory antibodies or to facilitate delivery of immunestimulating

genes, the technology has the potential capacity to improve the curative outcomes in both veterinary and human cancer cases. In conclusion, ECT should be an additional treatment of choice for canine MCT.

\section{Acknowledgements}

To all practitioner referring the cases for ECT procedure and to Dr Bernabo' N., University of Teramo, Italy for his valuable assistance for statistical analysis.

\section{References}

1. Marty M, Sersa G, Garbay JR, Gehl J, Collins CG, Snoj M, et al. Electrochemotherapy - an easy, highly e\#ective and safe treatment of cutaneous and subcutaneous metastases: results of ESOPE (European Standard Operating Procedures of Electrochemotherapy) study. European Journal of

Cancer Supplements 2006; 4: 3-13.

2. Spratt DE, Gordon Spratt EA,Wu S, DeRosa A, Lee $N Y$, Lacouture ME, et al. E\&cacy of skin-directed therapy for cutaneous metastases from advanced cancer: a meta-analysis. Journal of Clinical Oncology 2014; 32: 3144-3155.

3. Cemazar M, Tamzali Y, Sersa G, Tozon N, Mir LM, Miklavcic $D$, et al. Electrochemotherapy in veterinary oncology. Journal of Veterinary Internal

Medicine 2008; 22: 826-831.

4. Tello M, Oliveira L, Parise O, Buzaid AC, Oliveira $\mathrm{RT}$, Zanella R, et al. Electrochemical therapy to treat cancer (in vivo treatment). Conference Proceeding:

Annual International Conference of the IEEE Engineering in Medicine Biology Society 2007; 2007: 
3524-3527.

5. Sersa G."e state-of-the-art of

electrochemotherapy before the ESOPE study; advantages and clinical uses. European Journal of

Cancer Supplements 2006; 4: 52-59.

6. Spugnini EP, Baldi F, Mellone $P$, Feroce $F$, D'Avino $A$, Bonetto $F$, et al. Patterns of tumor response in canine and feline cancer patients treated with electrochemotherapy: preclinical data for the standardization of this treatment in pets and humans. Journal of Translational Medicine 2007; 5:

48.

7. Mali B, Jarm T, Snoj M, Sersa G and Miklavcic D. Antitumor e\#ectiveness of electrochemotherapy: a systematic review and meta-analysis. European Journal of Surgical Oncology 2013; 39: 4-16. 8. "amm DH, Vail DM. Mast Cell Tumours. In: Withrow SJ, MacEwen EG, editors. Small animal clinical oncology, 4th edn., Philadelphia,WB Saunders Co, 2007. p. 402-424.

9. Séguin B, Besancon MF, McCallan JL, Dewe LL, Tenwolde MC, Wong EK, et al. Recurrence rate, clinical outcome, and cellular proliferation indices as prognostic indicators a\$̦er incomplete surgical excision of cutaneous grade II mast cell tumors: 28 dogs (1994-2002). Journal of Veterinary Internal Medicine 2006; 20: 933-940.

10. Michels GM, Knapp DW, DeNicola DB, Glickman

$\mathrm{N}$ and Bonney $\mathrm{P}$. Prognosis following surgical excision of canine cutaneous mast cell tumors with histopathologically tumor-free versus nontumor-free margins: a retrospective study of 31 cases. Journal of the American Animal Hospital Association 2002; 38: 458-466.

11. Tozon N, Kodre V, Sersa G and Cemazar M. E\#ective treatment of perianal tumors in dogs with 
electrochemotherapy. Anticancer Research 2005; 25:

839-845.

12. Spugnini EP, Vincenzi $B$, Baldi $F$, Citro $G$ and Baldi A. Adjuvant electrochemotherapy for the treatment of incompletely resected canine mast cell tumors.

Anticancer Research 2006; 26: 4585-4589.

13. Spugnini EP, Vincenzi B, Citro G, Dotsinsky I, Mudrov T and Baldi A. Evaluation of Cisplatin as an electrochemotherapy agent for the treatment of incompletely excised mast cell tumors in dogs. Journal of Veterinary Internal Medicine 2011; 25:

407-411.

14. Kodre V, Cemazar M, Pecar J, Sersa G, Cor A and

Tozon N. Electrochemotherapy compared to surgery for treatment of canine mast cell tumours.

In Vivo 2009; 23: 55-62.

15. Patnaik AK, EhlerWJ and MacEwen EG. Canine cutaneous mast cell tumor: morphologic grading and survival time in 83 dogs. Veterinary Pathology 1984; 21: 469-474.

16. London CA,"amm DH. Mast Cell tumours. In: Withrow SJ, Vail DM, Page RL, editors. Withrow \&

MacEven's Small Animal Clinical Oncology, 5th edn., St. Louis, Elsevier Saunders, 2013. p. 335-355. 17. Krick EL, Billings AP, Shofer FS, Watanabe $S$ and Sorenmo KU. Cytological lymph node evaluation in dogs with mast cell tumours: association with grade and survival. Veterinary and Comparative Oncology 2009; 7: 130-138.

18. Romansik EM, Reilly CM, Kass PH, Moore PF and London CA. Mitotic index is predictive for survival for canine cutaneous mast cell tumors. Veterinary

Pathology 2007; 44: 335-341.

19. Gehl J, Sorensen TH, Nielsen K, Raskmark P, Nielsen SL, Skovsgaard T, et al. In vivo electroporation of skeletal muscle: threshold, 
e\&cacy and relation to electric !eld distribution. Biochimica et Biophysica Acta 1999; 1428: 233-240. 20. Gehl J. Electroporation: theory and methods, perspectives for drug delivery, gene therapy and research. Acta Physiologica Scandinavica 2003; 177: 437-447.

21. Mir LM. Bases and rationale of the electrochemotherapy. European Journal of Cancer, Suppl 2006; 4:

38-44.

22. Froudarakis $M$, Hatzimichael E, Kyriazopoulou L, Lagos K, Pappas P, Tzakos AG, et al. Revisiting bleomycin from pathophysiology to safe clinical use. Critical Reviews in Oncology/Hematology 2013;

87: 90-100.

23. Cadossi R, Ronchetti M and Cadossi M. Locally enhanced chemotherapy by electroporation: clinical experiences and perspective of use of electrochemotherapy.

Future Oncology 2014; 10: 877-890. 24. Suzuki DO, Anselmo J, de Oliveira KD, Freytag JO, Rangel MM, Marques JL, et al. Numerical model of dog mast cell tumor treated by electrochemotherapy.

Arti!cial Organs 2015; 39: 192-197.

25. Kiupel M,Webster JD, Bailey KL, Best S, DeLay J, Detrisac CJ, et al. Proposal of a 2-tier histologic grading system for canine cutaneous mast cell tumors to more accurately predict biological behavior. Veterinary Pathology 2011; 48: 147-55.

26. Webster JD, Yuzbasiyan-Gurkan V, Miller RA, Kaneene JB and Kiupel M. Cellular proliferation in canine cutaneous mast cell tumors: associations

with c-KIT and its role in prognostication.

Veterinary Pathology 2007; 44: 298-308.

27. O'Brien MA, Power DG, Clover AJ, Bird B, Soden DM and Forde PF. Local tumour ablative therapies: opportunities for maximising immune engagement 
and activation. Biochimica et Biophysica Acta 2014;

1846: 510-523.

28. Galon J, Mlecnik B, Bindea G, Angell HK, Berger A, Lagorce $C$, et al. Towards the introduction of the 'Immunoscore' in the classi!cation of malignant tumours. Journal of Pathology 2014; 232: 199-209. 29. FridmanWH, Dieu-Nosjean MC, Pages F, Cremer

I, Damotte D, Sautes-Fridman C, et al."e immune microenvironment of human tumors: general signi!cance and clinical impact. Cancer Microenvironment 2013; 6: 117-122.
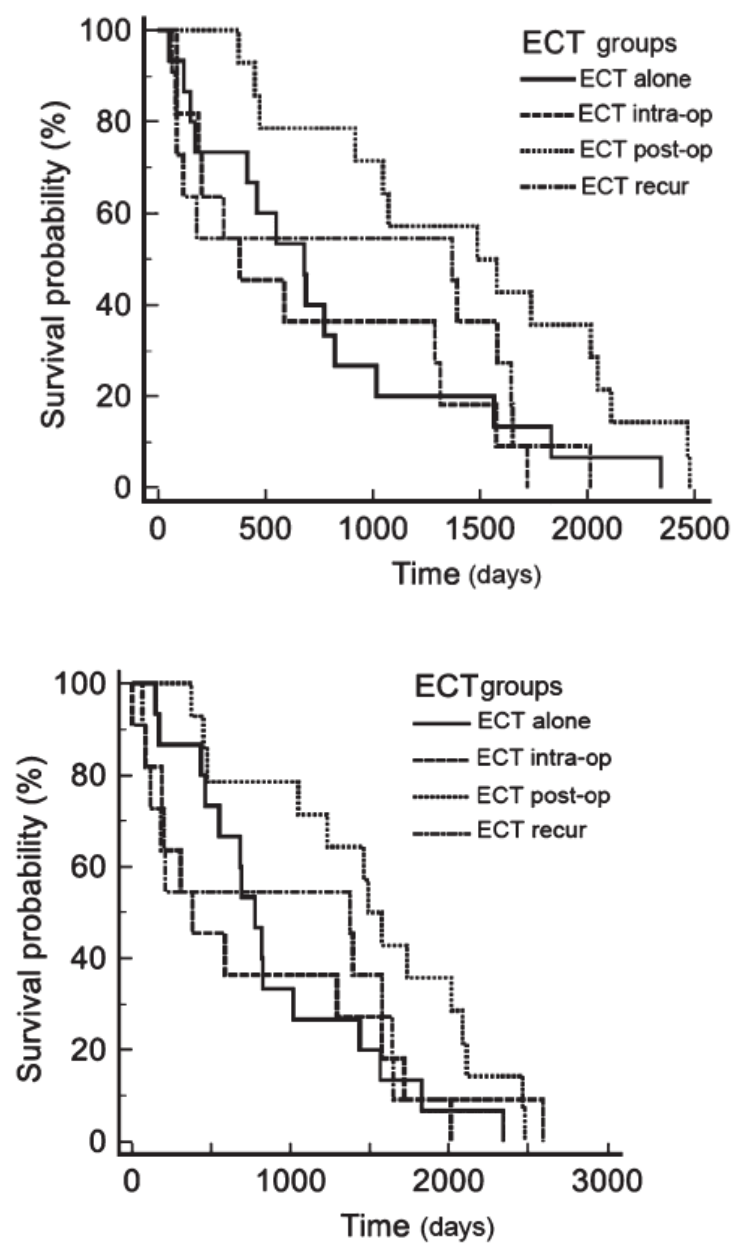